\title{
PHOTOELECTRIC OBSERVATIONS OF OCCULTATIONS
}

\section{IN JAPAN}

\author{
A. M. SINZI \\ Hydrographic Dept., Tokyo, Japan
}

\section{Geodetic Observations}

In Japan, photoelectric observations of occultations were made for the first time in 1951.

For about ten years after that, observations were made partly for geodetic purposes in order to determine the position of Japan with reference to islands in the Pacific, Phillippine and Taiwan.

The geodetic datum of Japan, which is currently adopted, suffers seriously from the vertical deflection. The amount of this deflection is supposed to be about $10^{\prime \prime}$ in latitude and $20^{\prime \prime}$ in longitude.

This project of occultation geodesy was, however, not so successful, because of the following two reasons: (1) Poor efficiency of data acquisition due to weather. We need good weather at two stations; the distance between them is usually over $1000 \mathrm{~km}$. (2) Difficulty of correction for the Moon's limb. For geodetic purposes, the atlas of the Moon's limb by Dr Watts is still insufficient.

Therefore, the project of occultation geodesy was replaced by satellite geodesy.

\section{Determination of ET}

On the other hand, observations at fixed stations have been made as routine since about 1952. At present, observations are carried out by two organizations:

(1) Tokyo Astron. Obs., $65 \mathrm{~cm}$ refractor.

(2) Hydrogr. Dept. 3 Observatories, $30 \mathrm{~cm}$ reflector each.

Photomultipliers are the usual ones, e.g. 1P21, EMI6094B, .... The Tokyo Astron. Obs. obtains $30-40$ observations in each year. The Hydrogr. Dept. obtained, until 1968 , about 80 photoelectric observations and about 300 visual observations. But in 1969 we obtained 120 photoelectric and 524 visual observations. The increase in number of successful events is due to the predictions provided by USNAO.

The probable error of $\Delta T$-evaluation is about 0.14 and that of $\Delta B$ is about $0^{\prime \prime} 10$.

The values of $\Delta T$, thus obtained, are sent to the observatories and institutes, which are concerned with time determination e.g. USNO, RGO, BIH, ..., and some members of IAU Commissions 4 and 31 .

However, in this evaluation of $\Delta T$, the most serious problem is the uncertainty of the geodetic datum of Japan. 


\section{Comparison of the Atlas of the Moon's Limb}

As a whole, Weimer's chart of the Moon's limb deviates from Watts by 0.43 in the direction of $345^{\circ}$ for eastern limb, and $0^{\prime \prime} .27$ in the direction of $296^{\circ}$ for western limb. The effect of this difference appears clearly in the evaluation of $\Delta T$, according to whether we employ Watts or Weimer's chart.

\section{Occultation of Mars by the Moon}

In January 1967, we observed the reappearance of an occultation of Mars by the Moon. Until that time we had never made photometric observation, and in this observation, we did not attach any filter or other photometric device to the telescope. Just before and after the occultation, some field stars were observed for comparison, and some main sequence members of Hyades were observed a few days later.

From these observations, we made a calibration curve between record and brightness, and various light curves were drawn for various slope of Moon's mountain.

The observed occultation curve agrees well with one of the calculated curves, for which slope of the Moon's mountain with respect to the Moon's mean surface is taken from Watt's charts for this occultation. $\mathrm{O}-\mathrm{C}$ in time for the reappearance of Mars' centre is $-2.5 \pm 0.3$ (p.e.). 